Research in Astron. Astrophys. 2010 Vol. 10 No. XX, 000-000

http://www.raa-journal.org http://www.iop.org/journals/raa

$\boldsymbol{R}$ esearch in

Astronomy and

Astrophysics

\title{
Measuring the beaming angle of GRB 030329 by fitting the rebrightenings in its multiband afterglow
}

\author{
Wei Deng, Yong-Feng Huang and Si-Wei Kong \\ Department of Astronomy, Nanjing University, Nanjing 210093, P. R. China; hyf@nju.edu.cn \\ Key Laboratory of Modern Astronomy and Astrophysics (Nanjing Univ), Ministry of Education, P. R. \\ China \\ Received [year] [month] [day]; accepted [year] [month] [day]
}

\begin{abstract}
Multiple rebrightenings have been observed in the multiband afterglow of GRB 030329. Especially, a marked and quick rebrightening occurred at about $\mathrm{t} \sim 1.2 \times 10^{5}$ s. Energy injection from late and slow shells seems to be the best interpretation for these rebrightenings. Usually it is assumed that the energy is injected into the whole external shock. However, in the case of GRB 030329, the rebrightenings are so quick that the usual consideration fails to give a satisfactory fit to the observed light curves. Actually, since these late/slow shells coast freely in the wake of the external shock, they should be cold and may not expand laterally. The energy injection then should only occur at the central region of the external shock. Considering this effect, we numerically re-fit the quick rebrightenings observed in GRB 030329. By doing this, we were able to derive the beaming angle of the energy injection process. Our result, with a relative residual of only $5 \%-10 \%$ during the major rebrightening, is better than any previous modeling. The derived energy injection angle is about 0.035 . We assume that these late shells are ejected by the central engine via the same mechanism as those early shells that produce the prompt gamma-ray burst. The main difference is that their velocities are much slower, so that they catch up with the external shock very lately and manifest as the observed quick rebrightenings. If this were true, then the derived energy injection angle can give a good measure of the beaming angle of the prompt $\gamma$-ray emission. Our study may hopefully provide a novel method to measure the beaming angle of gamma-ray bursts.
\end{abstract}

Key words: gamma-rays: bursts - ISM: jets and outflows

\section{INTRODUCTION}

GRB 030329, which is one of the brightest gamma-ray bursts (GRBs), has a fluence of about $1.18 \times$ $10^{-4}$ ergs $\cdot \mathrm{cm}^{-2}$ (Ricker et al. 2003; Vanderspek et al. 2004). It is also very close to us. The redshift is $\mathrm{z}=0.168$ (Greiner et al. 2003). At the same time, GRB 030329 is unambiguously confirmed to be associated with supernova (Hjorth et al. 2003; Stanek et al. 2003; Matheson et al. 2003). Because of these important characteristics, this GRB has attracted a lot of attentions. Numerous and detailed multiband afterglow observations have been accumulated. Multiple rebrightenings were observed in the afterglow of GRB 030329. Especially, a quick and marked rebrightening occurred at about $\mathrm{t} \sim 1.2 \times 10^{5} \mathrm{~s}$.

Several models have been proposed to explain the quick and marked rebrightenings. (i) Densityjump model: if the external shock encounters a sudden density variance of the circum-burst medium, the emission of the afterglow may be enhanced temporarily Wang \& Loeb 2000; Lazzati et al. 2002; 
Nakar \& Piran 2003; Dai \& Wu 2003; Tam et al. 2005). But for GRB 030329, it has been argued that the density-jump model could not produce the observed rebrightenings (Nakar \& Piran 2003; Willingale et al. 2004; Piran et al. 2004; Huang et al. 2006). (ii) The two-component jet model is another kind of choice (Berger et al. 2003). But Huang et al. (2006) made a detailed numerical calculation and found that although the two-component jet model could basically reproduce the overall R-band light curve, it was unable to explain the steep rebrightenings. (iii) Willingale et al. (2004) discussed a possibility that the rebrightening might be due to a supernova component, but their model is unlikely to produce multiple rebrightenings.

Actually, the energy-injection model seems to be the best explanation for the steep rebrightenings (Huang et al. 2006). Granot et al. (2003) have made a general analysis on this interpretation and Huang et al. (2006) presented a detailed numerical study. In Huang et al.'s study, it is interesting to note that although the fitting result was much better than previous models, the theoretical rebrightenings still could not be as steep as observations. This problem was probably caused by their simple assumption that the energy carried by the late (and initially slower) shells was injected into the whole external shock homogeneously. In reality, it was probable that the late/slow shells, which coast freely in the wake of the previous external shock, should be cold and do not expand laterally, as illustrated by Granot et al. (2003). So when the late/slow shells catch up with the external shock, the energy should only be injected into the central region of the external shock. We call this scenario as a localized energy-injection scenario. We conjecture that the energy-injection angle can be derived from the rapidness of the observed rebrightenings. Since these late/slow shells basically may have the same origin as those early shells that produce the internal shocks and the main GRB, we argued that this method could be used to hint the degree of collimation in GRBs. This will be a novel way to measure the beaming angle of GRBs, in addition to the traditional jet-break timing method.

In this paper, we will try to reproduce the multiband afterglow light curves of GRB 030329 numerically, based on the localized energy-injection scenario. The structure of our paper is organized as follows. Section 2 is a detailed description of the model. In Section 3, we study the effects of various parameters on the rapidness of the rebrightening. In Section 4, we simulate the observed multiband afterglow light curves of GRB 030329 in the framework of our model. Section 5 presents a brief discussion and the conclusion.

\section{LOCALIZED ENERGY-INJECTION}

According to the standard fireball model, when the fireball plows through the circum-burst medium, it will produce a strong blastwave that accelerates the swept-up electrons (Piran 1999; van Paradijs et al. 2000; Mészáros 2002; Zhang \& Mészáros 2004; Piran 2005). The afterglow is produced by these accelerated electrons. In this study, we will only consider the synchrotron radiation from electrons, although inverse Compton scattering may also play a role in some cases (Wei \& Lu 2000; Sari \& Esin 2001). The detailed condition of afterglow is quite complicated. For instance, the blastwave may evolve from highly radiative regime (the radiation efficiency $\epsilon=1)$ to adiabatic regime $(\epsilon=0)$. The blastwave will experience the transition from ultrarelativistic phase to Newtonian phase. Meanwhile, the effect of lateral expansion of the jet, the condition of the circum-burst medium (homogeneous or wind-like) and the equal arrival time surface effect (Waxman 1997; Sari 1998; Panaitescu \& Mészáros 1998) are also important factors which influence the evolution of the afterglow. In order to reproduce the complicated behavior of the afterglow of GRB 030329, all these factors should be considered. For this purpose, numerical evaluation is more efficient than analytical method. Huang et al. (1999) and Huang \& Cheng (2003) have developed an effective method which can easily take into account all the complex conditions addressed above. Recently, van Eerten et al. (2010) developed a more accurate code. But since Huang et al.'s code is more convenient for numerical solution, we will use their code for the current study.

To fit the overall multiband afterglow of GRB 030329, we basically need a two-component jet (Berger et al. 2003): a narrow jet with a small opening angle $\left(\theta_{0, \mathrm{n}}\right)$ and a very large initial Lorentz factor $\left(\gamma_{0, n}\right)$, and a wide jet with a relatively larger opening angle $\left(\theta_{0, \mathrm{w}}\right)$ but with a smaller initial 
Lorentz factor $\left(\gamma_{0, \mathrm{w}}\right)$. These two components are coaxial, and both of them may expand laterally when they push through the circum-burst medium. The narrow jet can produce the observed prompt GRB emission and the early afterglow, while the wide jet can contribute significantly to the late afterglow. In our localized energy-injection model, the key ingredient is the late/slow shells that play the role of energy-injection. We conjecture that these shells should be launched by the central engine via the same mechanism as the narrow jet component. The only difference is that they are launched slightly later, and most importantly, with much lower velocities. They catch up with the external shocks very lately, so that they could only manifest as energy-injections and rebrightenings in the afterglow, but not prompt gamma-ray emission. It is then reasonable to assume that the half opening angle of these late/slow shells should be similar to that of the narrow jet, i.e. $\theta_{0, n}$.

Before encountering the external shock, these late/slow shells move in the wake of the external shock. Since the circum-burst medium has been swept-up by the external blastwave, the wake should be very clean. It means that the movement of the late/slow shells should be nearly constant. So these shells must be cold and will not expand laterally. When they finally catch up with the external shock, energy injection will only occur at the central region of the GRB remnant, with the involved half opening angle (i.e., the energy-injection angle) still being $\theta_{0, n}$. In Figure 1, we present a schematic illustration of the scenario.

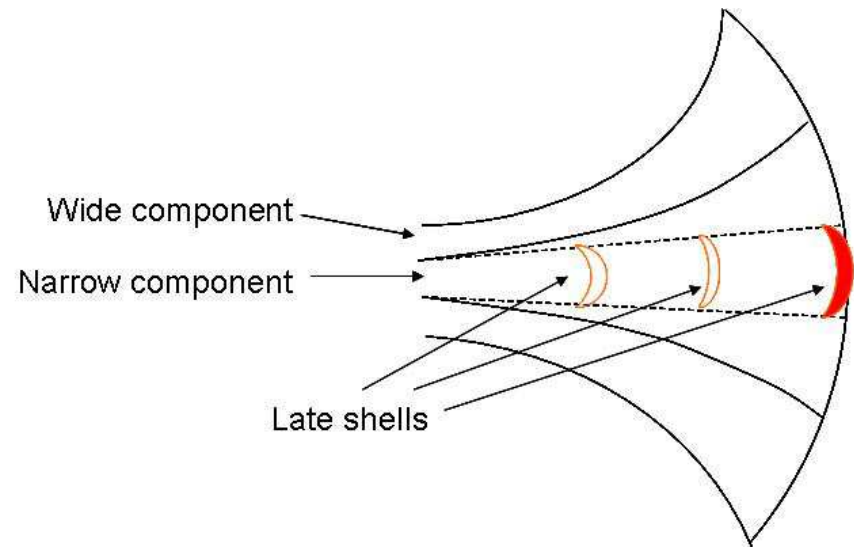

Fig. 1 A schematic illustration of the localized energy-injection process. The outer solid curves represent the wide component. The middle solid curves represent the narrow component. Both the wide and the narrow components can expand laterally. The inner dashed straight lines represent the trace of the late/slow shells, which coast freely and experience no lateral expansion before catching up with the external shock front. The half opening angle of the late/slow shells is assumed to equal to that of the narrow jet component, since they are most likely be of the same origin.

\section{RAPIDNESS OF THE REBRIGHTENING}

An energy-injection can naturally lead to a rebrightening in the afterglow. In the case of a localized energy-injection, the rebrightening should be much steeper as compared to a usual homogeneous energyinjection. A few parameters may affect the rapidness of the rebrightening, such as the number density of the circum-burst medium $(n)$, the half opening angle of the late/slow shells $\left(\theta_{0, \mathrm{n}}\right)$, the energy fraction of electrons with respect to protons $\left(\epsilon_{\mathrm{e}}\right)$, the energy fraction of magnetic field with respect to protons $\left(\epsilon_{\mathrm{B}}^{2}\right)$, the power-law index of electron distribution function $(p)$, etc. Analytically, the evolution of the radius $(R)$ and Lorentz factor $(\gamma)$ of the external shock is $R \propto n^{-1 / 4} t^{1 / 4}, \gamma \propto n^{-1 / 8} t^{-3 / 8}$ Huang et al. 

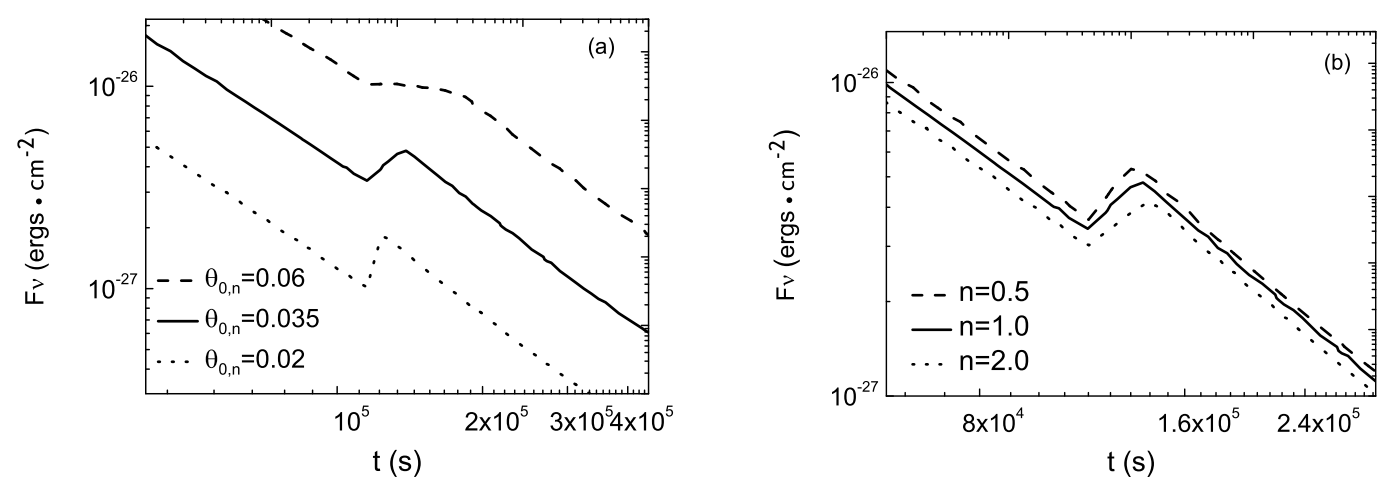

Fig. 2 The effects of the parameter $\theta_{0, n}$ (a) and $n$ (b) on the rebrightening caused by a localized energy-injection. In Panel (a), $\theta_{0, n}$ is in units of radian, and in Panel (b), $n$ is in units of $\mathrm{cm}^{-3}$. The values of other parameters are set constant in these calculations and are explained in the main text of Section 3.

1998). The angular timescale, i.e. the approximate duration of the rebrightening, can then be derived as $\Delta T \approx R_{\mathrm{inj}}\left(1-\cos \theta_{0, \mathrm{n}}\right) / c \approx R_{\mathrm{inj}} \theta_{0, \mathrm{n}}^{2} / 2 c \propto n^{-1 / 4} \theta_{0, \mathrm{n}}^{2} t_{\mathrm{inj}}^{1 / 4}$, where $R_{\mathrm{inj}}$ is the radius of the external

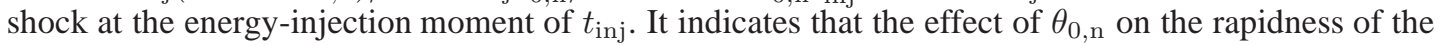
rebrightening is most striking, and the parameter $n$ only has a minor effect on the rebrightening, while the effects of other parameters are very insignificant.

We have numerically investigated the effects of the involved parameters. Our numerical results are consistent with the above analysis. In Fig. 2(a), we illustrate the effect of $\theta_{0, \mathrm{n}}$ on the afterglow light curve. In these calculations, we take $\epsilon_{\mathrm{e}}=0.1, \epsilon_{\mathrm{B}}^{2}=0.01, n=1 \mathrm{~cm}^{-3}, p=1.9$, and evaluated $\theta_{0, \mathrm{n}}$ as 0.02 , 0.035 , and 0.06 respectively. It is quite clear that a small energy-injection angle can lead to a very rapid rebrightening, and a large injection angle can make the rebrightening very smooth. It strongly hints us that we could use the rapidness of the observed rebrightening to derive the energy-injection angle.

As a comparison, we illustrate in Fig. 2(b) the effect of $n$ on the afterglow light curve. Again we have taken $\epsilon_{\mathrm{e}}=0.1, \epsilon_{\mathrm{B}}^{2}=0.01, p=1.9$, and $\theta_{0, \mathrm{n}}=0.035$, but let $n$ vary between $0.5 \mathrm{~cm}^{-3}$ and $2 \mathrm{~cm}^{-3}$. We see that the effect of $n$ on the rapidness of the rebrightening is much weaker.

In short, we find that the rapidness of the rebrightening is primarily determined by the energyinjection angle (i.e. the initial beaming angle). We suggest that we could use the observed afterglow rebrightenings to measure the beaming angle of GRBs. This is a novel and hopeful method to constrain the collimation degree of GRBs, independent to the conventional jet-break timing method.

\section{APPLICATION TO GRB 030329}

In this section, we use the localized energy-injection model to explain the multiband afterglow light curves of GRB 030329. Special attention will be paid to the remarkable rebrightening at $\mathrm{t} \sim 1.2 \times 10^{5}$ s. The observed data of optical afterglow are provided by Lipkin et al. (2004). However, the original data include the contribution from the host galaxy (Gorosabel et al. 2005) and the underlying supernova (Zeh et al. 2004, 2005). They are also contaminated by the extinction of our Galaxy (Schlegel et al. 1998). The pure optical afterglow light curves of GRB 030329 are available only when these contaminations are corrected for. We will use these pure afterglow light curves as the final template. The observed date of radio afterglow can be found in Berger et al. (2003). The effect of synchrotron self-absorption is considered in our calculations.

To fit the overall multiband afterglow of GRB 030329, we basically need a two-component jet model (Berger et al. 2003). The central narrow jet component can account for the prompt GRB emission and 
the early afterglow, while the wide jet component can account for the late afterglow. The observed multiple rebrightenings are then produced by a few energy-injections. In our framework, the energyinjection is caused by the late/slow shells ejected from the central engine. These shells should have the same origin as that of those early shells which generate the prompt GRB emission via internal shocks, except that they are ejected in a slightly late period and with much lower velocities. It is then natural to assume that the initial half opening angle of these shells equal to that of the early shells (i.e., the narrow jet component). The collision between a late shell and the external shock is very quick. We can approximate it as an instant energy-injection process (Rees \& Mészáros 1998; Kumar \& Piran 2000; Sari \& Mészáros 2000; Piran et al. 2004; King et al. 2005).

To fit the observed multiple rebrightenings, we find that at least 5 energy-injections are needed. After correctting for the cosmological redshift, the injection time (and the amount of energy needed) is $1.1 \times 10^{4} \mathrm{~s}\left(0.16 E_{\mathrm{n}, \text { iso }}\right), 1.0 \times 10^{5} \mathrm{~s}\left(0.15 E_{\mathrm{n}, \text { iso }}\right), 1.8 \times 10^{5} \mathrm{~s}\left(0.09 E_{\mathrm{n}, \text { iso }}\right), 2.2 \times 10^{5} \mathrm{~s}\left(0.04 E_{\mathrm{n}, \text { iso }}\right)$, and $3.6 \times 10^{5} \mathrm{~s}\left(0.06 E_{\mathrm{n} \text {,iso }}\right)$, respectively, where $E_{\mathrm{n} \text {,iso }}$ is the isotropic-equivalent kinetic energy of the narrow component. In Figs. 3 and 4, we illustrate our best fit to the multiband afterglow of GRB 030329. In these calculations, the initial half opening angle, the initial Lorentz factor and the isotropic-equivalent kinetic energy of the wide jet component are taken as $\theta_{0, \mathrm{w}}=0.2, \gamma_{0, \mathrm{w}}=50$, and $E_{\mathrm{w} \text {, iso }}=3.4 \times 10^{53}$ ergs. Correspondingly, the parameters of the narrow jet component are $\theta_{0, \mathrm{n}}=0.035, \gamma_{0, \mathrm{n}}=200$, and $E_{\mathrm{n}, \text { iso }}=2.0 \times 10^{53} \mathrm{ergs}$. The common parameters of the two components are $\epsilon_{\mathrm{e}}=0.1, \epsilon_{\mathrm{B}}^{2}=0.01, n=1$ $\mathrm{cm}^{-3}, p=1.9$. The luminosity distance is taken as $D_{\mathrm{L}}=0.8 \mathrm{Gpc}$. Note that we have assumed a $p$ value smaller than 2. Although such a small $p$ is not common for other events, it is still possible in GRBs (Dai \& Cheng 2001).

In Fig. 3, we see that the overall R-band afterglow light curve can be well explained. Especially, the observed rapid rebrightenings can be satisfactorily accounted for. Taking the most obvious rebrightening observed at $\sim 1.2 \times 10^{5} \mathrm{~s}$ as an example, this rebrightening is so rapid that all previous studies failed to present a satisfactory explanation. For example, in the study by Huang et al. (2006), since the energy was assumed to be homogeneously injected into the whole external shock, the theoretical rebrightening is then much slower than observations, and the relative residual is generally on a level of $\sim 20 \%$ at the rebrightening moment. In our current study, the rapidness problem is satisfactorily resolved. The relative residual is only about 5\% - 10\% during the rebrightening (see the bottom panel of Fig. 4).

Fig. 4 shows that most of the multiband afterglow light curves can be satisfactorily reproduced. The most obvious deviation appears in the $4.86 \mathrm{GHz}$ light curve. Our theoretical flux is lower than the observed intensity after $\sim 2 \times 10^{7} \mathrm{~s}$. Note that at this late stage, the decay of the observed $4.86 \mathrm{GHz}$ flux density becomes very slow and the light curve becomes flatter. It is possible that the contribution from the host galaxy begins to emerge from this point on. However, it is also possible that the structure of the ejecta in GRB 030329 were much more complicated than what we have considered here. In fact, Van der Horst et al. (2005) have argued that additional component is necessary to account for the excess of the observed flux at late stages. Finally, radio afterglow is subjected to scintillation due to scattering of interstellar medium (Frail et al. 2003). This may also explain part of the deviations, especially at early stages. Here, since our interest is mainly on the earlier optical rebrightenings, we suspend from extending our discussion to more of the above details.

Many other authors have studied the afterglow of GRB 030329 (Berger et al. 2003; Granot et al. 2003; Huang et al. 2006; Gao \& Dai 2010). It is interesting to note that the derived parameters vary from one group to another. For example, the suggested beaming angle of the narrow jet is 0.05 by Huang et al. (2006) and Gao \& Dai (2010), 0.07 by ?, and 0.09 by Berger et al. (2003). The suggested beaming angle of the wide jet is 0.3 by Berger et al. (2003), and 0.15 by Huang et al. (2006). In our current study, we take $\theta_{0, \mathrm{n}}=0.035$ and $\theta_{0, \mathrm{w}}=0.2$. Although these two parameters are again different from the above values, they are not unreasonable, and should be acceptable. Similarly, our other parameters are also in reasonable ranges. 


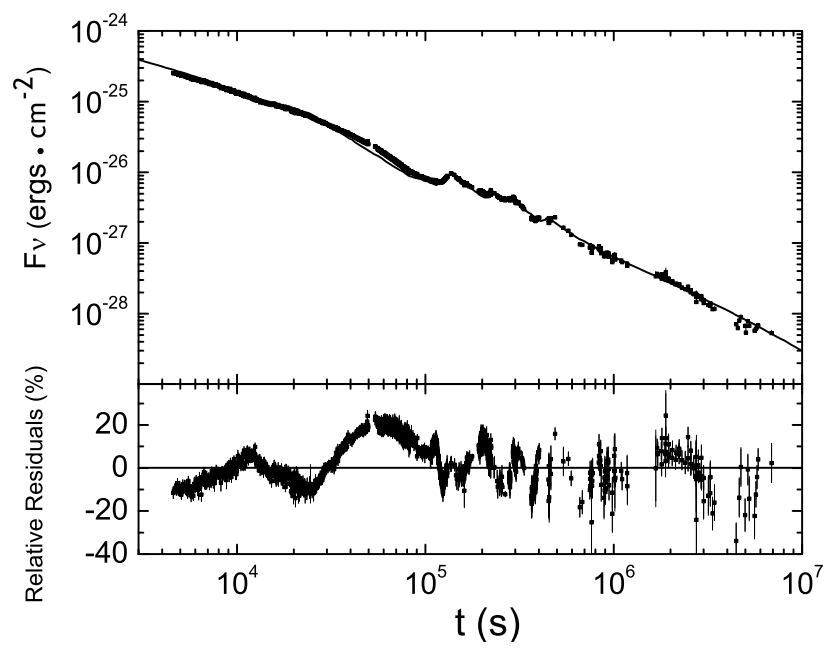

Fig. 3 Our best fit to the R-band afterglow light curve of GRB 030329, using the localized energy-injection model. Observed data points correspond to pure afterglow emission (Lipkin et al. 2004). The solid line corresponds to our best simulation with five major energyinjection processes which occur at $1.1 \times 10^{4} \mathrm{~s}, 1.0 \times 10^{5} \mathrm{~s}, 1.8 \times 10^{5} \mathrm{~s}, 2.2 \times 10^{5} \mathrm{~s}$, and $3.6 \times 10^{5} \mathrm{~s}$ respectively. The bottom panel gives the relative residual of the fit. See the main text in Section 4 for more details of this calculation.

\section{DISCUSSION AND CONCLUSION}

The rapid rebrightening is an interesting feature of the afterglow of GRB 030329. Conventional homogeneous energy-injection model cannot account for the rapidness of the observed rebrightenings. In this study, it is clearly shown that the localized energy-injection model can satisfactorily resolve the rapidness problem. By using a two-component jet model, together with 5 localized energy-injections, we can satisfactorily reproduce the overall and multiband afterglow light curves of GRB 030329. In our scenario, the narrow component gives birth to the prompt GRB emission and the early afterglow, while the wide component contributes significantly to the late afterglow. The energy-injections are induced by 5 late shells, which should be ejected by the central engine via the same or similar mechanism as the narrow jet component, but at a slightly later period and with much lower velocities. These shells coast freely in the empty environment after the external shock. They keep to be cold and do not experience any lateral expansion until they catch up with the external shock and inject their energies into the GRB remnant. The energy-injection is a prompt process, and most importantly, is a localized collision. It can lead to a very rapid increase in the afterglow brightness, barely subjected to the smearing of the equal arrival time surface effect.

The initial beaming angle is an important parameter of GRBs. It can provide important clues for the central engine (Cheng \& Lu 2001; Zhang 2007; Gao \& Dai 2010). According to our best fit, the energyinjection angle is about 0.035 in the case of GRB 030329. In our modeling, this angle also equals to the half opening angle of the narrow jet component. We also showed that the rapidness of the rebrightening is mainly controlled by the energy-injection angle. Other parameters, such as $\epsilon_{\mathrm{e}}, \epsilon_{\mathrm{B}}^{2}, p$, and $n$ only have minor or negligible effect. So, we suggest that the rapidness of the observed rebrightenings can basically be used to derive a measure of the beaming angle of GRBs. This is a novel and hopeful method, independent to the conventional jet-break timing method. Comparing with the jet-break timing method, the advantage of our method is that it does not rely on the assumption of the speed of lateral expansion, which itself is quite unclear currently. The impact of another factor, i.e. the density of the circum-burst 

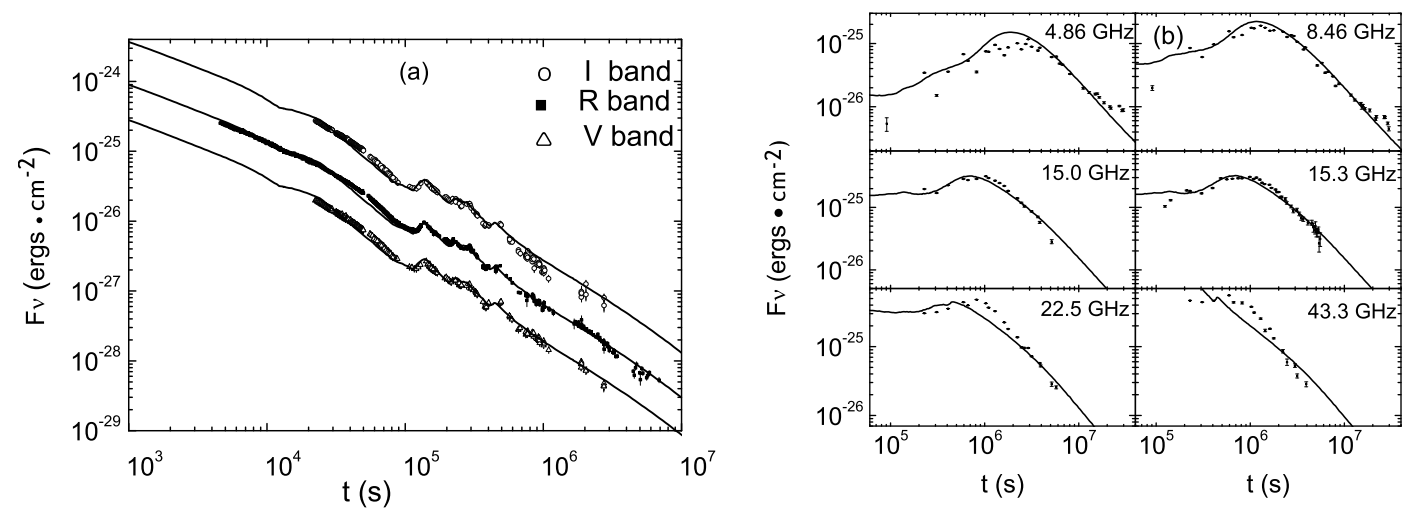

Fig. 4 Our best fit to the multiband afterglow light curves of GRB 030329 using the localized energy-injection model. Panel (a) corresponds to optical afterglow. The observed data points are derived from Lipkin et al. (2004). Panel (b) corresponds to radio afterglow. The observed data points are taken from Berger et al. (2003). Note that in Panel (a), the I and V band light curves have been shifted by -1.2 and +1.2 magnitude respectively, for clarity. See the main text in Section 4 for more details of this calculation.

environment, is also very week in our method. However, the main restriction of our method is that it is applicable only when rapid rebrightenings due to energy-injections were observed in the afterglow.

In our modeling of the five observed rebrightenings of GRB 030329, the beaming angles of the 5 energy-injections are set to be constant. The fitting results are good. It means that the ejecting angle of the central engine might not vary significantly over the active period. However, in the future, it is possible that we could find new examples which need to vary the energy-injection angle. Such information will also provide interesting constraints on the central engine. In our model, we have assumed that the energyinjection angle equals to the beaming angle of the narrow jet that produces the prompt GRB. Although this assumption is a natural hypothesis, it is still possible that in reality they might not equal, since the real central engine may be much more complicated as compared with our imagination. Even in this case, the energy-injection angle derived from observations still can give useful hints on the characteristics of the central engine.

In our current study, in order to get an acceptable fit to the overall and multiband afterglow of GRB 030329, we have adopted a two-component jet geometry. However, note that such a two-component configuration is not always necessary for localized energy-injection. For example, in other GRBs, it is quite possible that the prompt GRB jet may only have one component. This component can expand laterally during its deceleration, still leaving a relatively clean environment behind it. So, late shells will still coast freely after the external shock. They keep to be cold and will not experience sideways expansion before the final energy-injection.

For GRB 030329, in order to get an acceptable fit to the overall and multiband afterglow, we have adopted a $p$ value that is less than 2 . However, as stated in section $3, p$ does not influence the rapidness of the rebrightening. So $p<2$ is not necessary for the localized energy-injection model. In other GRBs, different $p$ can be adopted according to observations.

In many GRBs, multiple optical and/or X-ray flares are observed to be superposed on the early afterglow light curves. The rising and falling of these flares are even more rapid than that in the case of GRB 030329. Although the nature of these flares is still largely unknown, we suggest that useful information on the beaming angle of these flares can also be derived by fitting the rising and falling profiles. 
Acknowledgements We thank the anonymous referee for helpful comments and suggestions, and X. Wang for useful discussion. This work was supported by the National Natural Science Foundation of China (Grant No. 10625313) and the National Basic Research Program of China (973 Program, Grant 2009CB824800).

\section{References}

Berger, E., Kulkarni, S. R., Pooley, G., et al. 2003, Nature, 426, 154

Cheng, K. S., \& Lu, T. 2001, Chin J Astron Astrophys, 1, 1

Dai, Z. G., \& Cheng, K. S. 2001, ApJ, 558, L109

Dai, Z. G., \& Wu, X. F. 2003, ApJ, 591, L21

Frail, D. A., Yost, S. A., Berger, E., et al. 2003, ApJ, 590, 992

Gao, Y., \& Dai, Z. G. 2010, RAA, 10, 142

Gorosabel, J., Pérez-Ramírez, D., Sollerman, J., et al. 2005, A\&A, 444, 711

Granot, J., Nakar, E., \& Piran, T. 2003, Nature, 426, 138

Greiner, J., Peimbert, M., Estaban, C., et al. 2003, GCN Circ. 2020 (http://gen.gsfe.nasa.gov/gen/gcn3/2020.gcn3)

Hjorth, J., Sollerman, J., Møller, P., et al. 2003, Nature, 423, 847

Huang, Y. F., Dai, Z. G., Wei, D. M., Lu, T. 1998, MNRAS, 298, 459

Huang, Y. F., \& Cheng, K. S. 2003, MNRAS, 341, 263

Huang, Y. F., Cheng, K. S., \& Gao, T. T. 2006, ApJ, 637, 873

Huang, Y. F., Dai, Z. G., \& Lu, T. 1999, MNRAS, 309, 513

King, A., O'Brien, P. T., Goad, M. R., et al. 2005, ApJ, 630, L113

Kumar, P., \& Piran, T. 2000, ApJ, 532, 286

Lazzati, D., Rossi, E., Covino, S., et al. 2002, A\&A, 396, L5

Lipkin, Y. M., Ofek, E. O., Gal-Yam, A., et al. 2004, ApJ, 606, 381

Matheson, T., Garnavich, P. M., Stanek, K. Z., et al. 2003, ApJ, 599, 394

Mészáros, P. 2002, ARA\&A, 40, 137

Nakar, E., \& Piran, T. 2003, ApJ, 598, 400

Panaitescu, A., \& Mészáros, P. 1998, ApJ, 493, L31

Piran, T. 1999, Phys. Reports, 314, 575

Piran, T. 2005, Rev. Mod. Phys., 76, 1143

Piran, T., Nakar, E., \& Granot, J. 2004, in AIP Conf. Proc. 727, Gamma-Ray Bursts: 30 Years of Discovery, ed. E.

E. Fenimore \& M. Galassi (Melville: AIP), 181

Rees, M. J., \& Mészáros, P. 1998, ApJ, 496, L1

Ricker, G. R., et al. 2003, IAU Circ. 8101

Sari, R. 1998, ApJ, 494, L49

Sari, R., \& Esin, A. A. 2001, ApJ, 548, 787

Sari, R., \& Mészáros, P. 2000, ApJ, 535, L33

Schlegel, D. J., Finkbeiner, D. P., \& Davis, M. 1998, ApJ, 500, 525

Stanek, K. Z., Matheson, T., Garnavich, P. M., et al. 2003, ApJ, 591, L17

Tam, P. H., Pun, C. S. J., Huang, Y. F., \& Cheng, K. S. 2005, NewA, 10, 535

Van der Horst, A. J., Rol, E., Wijers, R. A. M. J., et al. 2005, ApJ, 634, 1166

Vanderspek, R., Sakamoto, T., Barraud, C., et al. 2004, ApJ, 617, 1251

Van Eerten, H. J., Leventis, K., Meliani, Z., Wijers, R. A. M. J., Keppens, R. 2010, MNRAS, 403, 300

van Paradijs, J., Kouveliotou, C., \& Wijers, R. 2000, ARA\&A, 38, 379

Wang, X. H. \& Loeb, A. 2000, ApJ, 535, 788

Waxman, E. 1997, ApJ, 491, L19

Wei, D. M., \& Lu, T. 2000, A\&A, 360, L13

Willingale, R., Osborne, J. P., O’Brien, P. T., et al. 2004, MNRAS, 349, 31

Zeh, A., Klose, S., \& Hartmann, D. H. 2004, ApJ, 609, 952

Zeh, A., Klose, S., \& Hartmann, D. H. 2005, in Conf. Proc. of the 22nd Texas Symposium on Relativistic Astrophysics, ed. P. Chen, E. Bloom, G. Madejski, \& V. Patrosian, eConf C041213, 2225 
Zhang, B. 2007, Chin J Astron Astrophys, 7, 1

Zhang, B., \& Mészáros, P. 2004, Int J Mod Phys A, 19, 2385 